\title{
Stationäre Behandlung depressiver Erkrankungen wohnortnah oder wohnortfern: Gibt es Unterschiede im Therapieergebnis?
}

\author{
Alice Diedrich ${ }^{a, b} \quad$ Heike Ewald ${ }^{c}$ \\ Ulrich Voderholzer ${ }^{\mathrm{b}, \mathrm{g}}$ \\ Gernot Langs $^{d}$ \\ a Klinikum der Ludwig-Maximilians-Universität München, München, Deutschland; \\ ${ }^{b}$ Schön Klinik Roseneck, Prien am Chiemsee, Deutschland; \\ 'Schön Klinik Zentrales Qualitätsmanagement, Prien am Chiemsee, Deutschland; \\ ${ }^{\mathrm{d}}$ Schön Klinik Bad Bramstedt, Bad Bramstedt, Deutschland; \\ e Schön Klinik Bad Staffelstein, Bad Staffelstein, Deutschland; \\ fSchön Klinik Bad Arolsen, Bad Arolsen, Deutschland; \\ g Klinikum der Albert-Ludwigs-Universität Freiburg, Freiburg i.Br., Deutschland
}

Elisabeth Rauh ${ }^{\mathrm{e}}$

Thomas Gärtner ${ }^{f}$

\section{Schlüsselwörter}

Wohnortfern · Wohnortnah · Distanz Wohnort-Klinik .

Depressive Erkrankungen

\section{Zusammenfassung}

Hintergrund: Seit der Psychiatrie-Enquete werden die Vorund Nachteile wohnortferner und wohnortnaher stationärer Behandlung psychisch Erkrankter kontrovers diskutiert. Ob die Distanz des Wohnorts zur Klinik Einfluss auf den Behandlungserfolg hat, wurde bisher jedoch nur in der stationären medizinischen Rehabilitation bei Patienten mit Abhängigkeitserkrankungen untersucht. Ziel der vorliegenden Studie ist deshalb die Untersuchung des Einflusses der Distanz zwischen Wohnort und Behandlungseinrichtung auf den Behandlungserfolg depressiver Patienten. Patienten und Methoden: Hierzu wurden die Routinedaten von 1959 stationär behandelten Patienten mit der Diagnose einer depressiven Erkrankung analysiert. Depressivität, Ängstlichkeit und Somatisierungsbeschwerden wurden zur Erfassung des Therapieerfolgs bei Aufnahme, bei Entlassung und katamnestisch 6 Monate später mit dem Patient Health Questionnaire erfasst. Ergebnisse: Die Ergebnisse zeigen, dass die Distanz zwischen Wohnort und Klinik die Veränderung der depressiven, ängstlichen und somatoformen Symptomatik während und nach der Behandlung nicht moderiert. Auch zeigen sich keinerlei Zusammenhänge zwischen der Distanz Wohnort-Klinik und den Symptomveränderungen zwischen Aufnahme und Katamnese. Schlussfolgerungen: Empirische Belege für Unterschiede im Outcome in Abhängigkeit von der Distanz zwischen Wohnort und Klinik liegen nicht vor. Zukünftig sollte deshalb der Einfluss der inhaltlichen Aspekte wohnortnaher und -ferner Behandlung, wie der Spezialisierungsgrad der Einrichtung sowie die Umsetzung individueller Nachsorgemaßnahmen, auf den Erfolg stationärer Therapien systematisch untersucht werden.

\section{Keywords}

Close to home - Far away from home .

Distance residence-clinic - Depressive disorders

\section{Summary}

Inpatient Treatment of Individuals Suffering from Depressive Disorders Close to or Far Away from Home: Are There Differences in Treatment Outcome?

Background: Advantages and disadvantages of inpatient treatment for mental disorders in facilities located either close to or distant from the patients' homes have been controversially discussed since the Psychiatry Enquiry. However, the question whether the distance between residence and clinic influences the treatment outcome has so far only been examined in substance-dependent patients in residential medical rehabilitation. Therefore, in the present study, we aimed to investigate potential effects of the distance between residence and clinic on the treatment outcome in depressive patients. Patients and Methods: For this purpose, routine data of 1,959 clinically depressed inpatients were analyzed. Using the Patient Health Questionnaire, depression, anxiety, and somatization were assessed at admission, discharge, and the 6-month follow-up to analyze the treatment outcome. Results: The findings demonstrate that the distance between the residence and the treatment facility does not moderate depressive, anxious, and somatoform symptom changes during and after treatment. Moreover, the distance between clinic and residence and the symptom improvement from admission until follow-up do not correlate significantly. Conclusions: Hence, there is no empirical evidence showing that the distance between the residence and the treatment facility influences the treatment outcome. Therefore, future research should focus on the impact of specific treatment qualities close to or far away from home, like the facility's degree of specialization and the implementation of individualized aftercare.

\section{KARGER}

(C) 2016 S. Karger GmbH, Freiburg 


\section{Hintergrund}

In der sogenannten Psychiatrie-Enquete von 1975 wurden gravierende Mängel in der psychiatrischen Versorgung psychisch erkrankter Menschen festgestellt [Arolt et al., 2011]. Unter anderem wurde kritisiert, dass Betroffene immer noch in großen Landeskrankenhäusern fernab von ihrem Wohnort behandelt wurden. Da damals die Besuchsmöglichkeiten aufgrund schlechterer Transportmöglichkeiten im Vergleich zu heute deutlich reduziert waren und die Behandlungen wesentlich länger andauerten, kam es oft zu einer sozialen Desintegration der Patienten [Hell, 2002], was wiederum die langfristige Genesung der Patienten erschwerte. Um diesen und weitere Mängel der stationären Versorgungssituation psychisch Erkrankter in Deutschland zu beheben, wurde unter anderem beschlossen, sich die Ausweitung einer gemeinde- bzw. wohnortnahen stationären Behandlung zum Ziel zu setzen [Arolt et al., 2011; Fritze et al., 2001].

Bis heute sind sich Praktiker und Wissenschaftler jedoch uneinig, ob die Nähe der stationären Behandlungsinstitution zum Wohnort tatsächlich einen Einfluss auf den Behandlungserfolg psychisch erkrankter Menschen hat [Fritze et al., 2001; Hohagen, 2002, 2007]. Klare Definitionen für wohnortnahe oder -ferne Versorgung liegen bisher nicht vor, in der Regel werden beide Versorgungsformen jedoch mit bestimmten inhaltlichen Qualitäten in Verbindung gebracht [Fritze et al., 2001; Hohagen, 2002, 2007]. Als Qualitäten einer wohnortnahen Versorgung werden insbesondere die Aufrechterhaltung der Einbindung der Patienten in ihr soziales und familiäres Netzwerk und die daraus resultierende bessere soziale Unterstützung genannt [Hell, 2002; Hohagen, 2002, 2006]. Besuche durch Angehörige und Bekannte seien leichter realisierbar. Vor dem Hintergrund dessen, dass Einsamkeit einen Risikofaktor für depressive Erkrankungen und Suizidalität darstellt und die Prognose bei Angststörungen und psychotischen Störungen verschlechtert, kann angenommen werden, dass eine gute soziale Einbindung ein wesentlicher Faktor bei der Genesung psychisch erkrankter Menschen ist [Hell, 2002]. Darüber hinaus kann man argumentieren, dass bei einer wohnortnahen Behandlung die Eingliederung in den häuslichen Alltag im Anschluss an die Behandlung durch befristete Probeaufenthalte während der Behandlung erleichtert wird. Außerdem kann man davon ausgehen, dass bei der wohnortnahen Behandlung bestimmte Interventionen, wie z.B. Expositionsübungen, besser im häuslichen Umfeld erprobt werden können. Auch interaktionelle Probleme mit Angehörigen können mittels Familien-, Eltern- und/oder Paargesprächen im Rahmen der wohnortnahen Versorgung vermutlich besser thematisiert werden. Schließlich werden die größeren Chancen auf eine ambulante Anschlussbehandlung durch wohnortnah gut vernetzte Behandlungsinstitutionen als ein qualitativer Vorteil wohnortnaher Behandlung angeführt [Fritze et al., 2001; Hohagen, 2007]. Durch prompte ambulante Weiterbehandlungen könne bestmöglich an stationäre Therapieerfolge angeschlossen werden, was die Genesung von psychisch erkrankten Menschen beschleunigen könne. Bei einer wohnortfernen Behandlung seien diese Vorteile schwerer realisierbar.
Auf der anderen Seite werden auch für die wohnortferne stationäre Behandlung spezifische vorteilhafte qualitative Aspekte angeführt. Genannt werden in diesem Kontext vor allem die Möglichkeit einer diskreten Behandlung sowie die Möglichkeit einer Behandlung in einem beschützten Rahmen bei einem potenziell destruktiven privaten Umfeld [Funke und Missel, 2009]. Nicht selten wird sowohl von Betroffenen als auch von einweisenden Kollegen eine wohnortferne Behandlung explizit gewünscht. Beispielsweise kann es bei Abhängigkeitserkrankungen sinnvoll sein, Patienten zumindest in der Anfangsphase der Abstinenz vor einem möglicherweise ebenso suchtgefährdeten Umfeld zu schützen, um das Rückfallrisiko zu reduzieren. Hierin kann ein wesentlicher therapeutischer Wirkfaktor enthalten sein, da eine über längere Zeit anhaltende Abstinenz das Selbstwirksamkeitserleben der Patienten erhöhen kann und somit die Chancen auf eine langfristige Abstinenz [Zingg et al., 2009]. Zuletzt bieten wohnortferne, meist ländlich gelegene größere Fachkliniken oft stark individualisierte und störungsspezifische Behandlungen an, was in den wohnortnahen, oft städtisch gelegenen, weniger spezialisierten Behandlungsinstitutionen häufig aufgrund der Größe der Institutionen nicht so leicht gewährleistet werden kann [Fritze et al., 2001].

Von einer theoretischen Perspektive aus betrachtet scheinen also sowohl die wohnortferne als auch die wohnortnahe Behandlung spezifische vorteilhafte qualitative Aspekte aufzuweisen. In der vorliegenden Studie soll in einem ersten Schritt geprüft werden, ob die Distanz zwischen Wohnort und Klinik einen Einfluss auf den Therapieerfolg hat, und zwar unter der Annahme, dass die häufig diskutierten inhaltlichen Aspekte in der jeweiligen Bedingung (wohnortnah vs. wohnortfern) leichter realisierbar sind. Zum Einfluss der Distanz zwischen Klinik und Wohnort auf das Therapieergebnis bei psychischen Erkrankungen wurde unseres Wissens bisher nur 1 Studie durchgeführt. Funke und Missel [2009] untersuchten den Einfluss der Entfernung auf die Art der Entlassung (planmäßig, vorzeitig) sowie die Effektivität der Behandlung (Abstinenz, Rückfall) in der stationären medizinischen Rehabilitation in einer Stichprobe von 958 Patienten mit einer Alkohol- und/oder Drogenabhängigkeit. Die Ergebnisse weisen darauf hin, dass es bei Suchtkrankten scheinbar keine Unterschiede in der Art der Entlassung oder in der Effektivität der Behandlung in Abhängigkeit von der Wohnortnähe gibt. Darüber hinaus liegen bisher jedoch keine empirischen Hinweise für die Überlegenheit einer der beiden Behandlungsoptionen hinsichtlich des Therapieergebnisses vor.

Neben Patienten mit Abhängigkeitserkrankungen werden insbesondere Patienten mit depressiven Erkrankungen häufig in Kliniken in ländlich gelegenen Gegenden fernab von städtischen Ballungszentren behandelt [Schulz et al., 2011]. Diese Betroffenen leiden unter einer hochprävalenten [Busch et al., 2013], oft komorbiden [Rohde et al., 1991] und rezidivierenden oder chronischen psychischen Erkrankung [Wittchen et al., 2010], die mit einer bedeutsamen Morbidität, Mortalität, Behinderung und emotionalen Belastung für sie selbst und ihre Familien verbunden ist [Wittchen et al., 2010; Lépine und Briley, 2011]. Vor diesem Hintergrund er- 
scheint es besonders bei dieser Stichprobe wichtig zu untersuchen, ob sich die Distanz zwischen Wohnort und Klinik auf den Behandlungserfolg auswirkt.

\section{Methode}

\section{Studiendesign und Setting}

Die vorliegende Studie basiert auf den Routinedaten der 7 Kliniken der Schön Klinik (Bad Arolsen, Bad Bramstedt, Bad Staffelstein, Berchtesgadener Land, Hamburg-Eilbek, Roseneck, Starnberger See). In den genannten Kliniken werden Patienten mit psychischen Erkrankungen vorwiegend mit Diagnosen der Kategorien F3-F6 nach ICD-10 (Internationale Klassifikation psychischer Störungen [Dilling et al., 1993]) behandelt, bei denen eine Indikation für eine stationäre Behandlung besteht. Patienten mit mittelschweren oder schweren depressiven Episoden, meist mit Resistenz gegen vorausgegangene ambulante Therapien, stellen dabei die größte Behandlungsgruppe dar. Die Behandlung erfolgt multimodal auf Basis der S3-Leitlinien/Nationalen Versorgungsleitlinien für Unipolare Depression [DGPPN et al., 2009] durch ein Team aus Ärzten, Psychologen, Fachtherapeuten und Pflegekräften (Co-Therapeuten). Alle Patienten, die in ausreichender Nähe zur Klinik wohnen (auch die in vollstationärer Behandlung), erhalten Paar-, Familien- oder Elterngespräche, wenn dies indiziert und vom Patienten gewünscht ist. Darüber hinaus werden während der wohnortnahen Behandlung bestimmte Interventionen, wie Expositionsübungen oder Alltagsstrukturierung, sowie die Wiedereingewöhnung in den Alltag bei Kurzaufenthalten im häuslichen Umfeld erprobt. Insgesamt behandeln alle eingeschlossenen Kliniken sowohl wohnortnah als auch wohnortfern.

\section{Messmethoden}

Soziodemografische, klinische und behandlungsspezifische Variablen wurden im Rahmen der Basisdokumentation der stationären Behandlung der Patienten erfasst. Die Distanz zwischen Wohnort und Behandlungseinrichtung wurde als Luftlinie über die Koordinaten der Postleitzahlen der Wohnorte berechnet. Darüber hinaus füllten die Patienten als Teil der stationären Routinediagnostik bei Aufnahme, Entlassung und katamnestisch 6 Monate nach Entlassung neben anderen Instrumenten die deutsche Version des Patient Health Questionnaire (PHQ-D) aus [Löwe et al., 2002]. Für die vorliegende Studie wurden die Daten der 3 Module Depressivität, Ängstlichkeit und Somatisierung des PHQ-D herangezogen, um das Therapieergebnis hinsichtlich einer Veränderung der depressiven, ängstlichen und somatoformen Beschwerden zu erfassen. Das Depressionsmodul PHQ-9 umfasst 9 Items (Items 2a-i), die den 9 Kriterien einer depressiven Episode im DSM-IV (Diagnostisches und Statistisches Manual Psychischer Störungen [APA, 2000]) entsprechen. Die Stärke der Beeinträchtigung durch die Symptome wird auf einer Skala von 0 («überhaupt nicht») bis 3 («beinahe jeden Tag») eingeschätzt. Aus den Antworten wird der Summenwert gebildet, der Werte zwischen 0 und 27 annehmen kann. Das Angstmodul des Generalized Anxiety Disorder Screener (GAD-7) besteht aus 7 Items ( $4 \mathrm{a}-\mathrm{g}$ ), die auf einer Skala von 0 («überhaupt nicht») bis 3 («beinahe jeden Tag») in ihrer Intensität eingeschätzt werden müssen. Somit ergibt sich ein Summenwert zwischen 0 und 21. Das Somatisierungsmodul PHQ-15 umfasst 13 Items, die die häufigsten somatoformen Beschwerden messen (Items 1a-m), sowie 2 Items aus dem Depressionsmodul (Items 2c, 2d; Schlafschwierigkeiten und Energielosigkeit). Das Ausmaß der Beeinträchtigung in Bezug auf die Items 1a-m wird auf einer Skala von 0 («nicht beeinträchtigt») bis 2 («stark beeinträchtigt») eingestuft. Da die Skalierung der Items des Depressionsmoduls von 0 bis 3 reicht, werden Antworten, die mit 3 kodiert werden, in 2 umkodiert. Somit ergibt sich für das Somatisierungsmodul insgesamt ein Summenwert zwischen 0 und 30 .

In einer deutschen Stichprobe fanden Gräfe et al. [2004] eine interne Konsistenz nach Cronbachs a von 0,88 für die Depressivitätsskala und von 0,79 für die Somatisierungsskala. Für die Angstskala liegen zu Cronbachs a nur Ergebnisse des englischsprachigen PHQ vor ( $\alpha=0,92$ [Spitzer et al., 2006]). RetestReliabilitäten liegen ebenso nur für den englischsprachigen PHQ vor und liegen beim Depressionsmodul zwischen ICC $=0,81$ und ICC $=0,96$ und beim Angstmodul bei ICC $=0,83$ (ICC $=$ Intraklassenkorrelation) [Spitzer et al., 2006; Löwe et al., 2004]. Die Konstruktvalidität der Depressionsskala des englischen PHQ kann als gut betrachtet werden, was durch die faktorenanalytische Bestätigung der Eindimensionalität des Konstrukts sowie durch kleine bis hohe Korrelationen mit anderen Depressionsmaßen und verschiedenen Indikatoren psychischer Gesundheit belegt wird [Kroenke et al., 2001; Titov et al., 2011]. Bei der englischsprachigen Angstskala des PHQ deuten sowohl die Bestätigung der Eindimensionalität des Konstrukts sowie signifikante Zusammenhänge mit Indikatoren funktioneller Einschränkung sowie Selbstwerterleben auf eine gute Konstruktvalidität hin [Spitzer et al., 2006; Löwe et al., 2008]. Für das Somatisierungsmodul liegen empirische Nachweise für eine gute konvergente Validität in Form von signifikanten Korrelationen mit dem allgemeinen Funktionsniveau, Krankheitstagen und symptombezogenen Schwierigkeiten vor [Kroenke et al., 2002].

\section{Stichprobe}

In die Studie eingeschlossen wurden Patienten 1) mit der Diagnose einer depressiven Erkrankung (F32.0-F32.8, F33.0-F33.9, F34.1) nach ICD-10, 2) mit einem Mindestalter von 18 Jahren, 3) mit einem Aufnahme- und Entlassungsdatum im Zeitraum von Januar 2013 bis Dezember 2013 sowie 4) mit vorliegenden PHQ-Daten bei Aufnahme und Entlassung. Hieraus ergab sich eine Ausgangsstichprobe von $\mathrm{N}=3259$ Patienten. Von 39,9\% dieser Patienten fehlte der PHQ bei Katamnese, sodass letztlich 1959 Probanden in die Analysen eingingen. Die Rücklaufquote der Katamnese lag dementsprechend bei 60,1\%. Patienten mit und ohne Katamnese unterschieden sich in Bezug auf verschiedene demografische (Geschlecht, Alter, Partner, Kinder, Bildungsstand), klinische (PHQ-Aufnahmewerte aller 3 Skalen, Komorbidität, Anzahl Vorbehandlungen) sowie behandlungsspezifische (Behandlungsdauer, antidepressive Begleitbehandlung) Variablen bei Aufnahme unter Anwendung eines Bonferroni-korrigierten Alpha-Levels von 0,004 lediglich hinsichtlich des Alters ( $\mathrm{t}(2658)=$ $-6,90 ; \mathrm{p}<0,001)$. Patienten ohne Katamnese waren jünger als die mit Katamnese. Die klinischen Diagnosen wurden von Psychologen, Psychotherapeuten und Ärzten unter Supervision erfahrener Fachärzte der Psychiatrie und Psychotherapie oder Psychosomatik gestellt. Die soziodemografischen, klinischen sowie behandlungsbezogenen Merkmale der Stichprobe können der Tabelle 1 entnommen werden.

\section{Analysen}

Die statistische Datenauswertung wurde mit SPSS (Statistical Package for the Social Sciences) Version 20.0 durchgeführt. Zur Untersuchung der Fragestellung, ob sich das Therapieergebnis gemessen an der Ausprägung der Depressivität, Ängstlichkeit und Somatisierung bei Entlassung oder beim Katamnesezeitpunkt 6 Monate später signifikant in Abhängigkeit von der Distanz der Klinik zum Wohnort unterscheidet, wurde im ersten Schritt aus der kontinuierlichen Variable Distanz zum Wohnort in Kilometern mittels Mediansplit eine neue, kategoriale Variable mit den 2 Stufen wohnortnah (0$134,52 \mathrm{~km})$ und wohnortfern (134,53-794,43 km) gebildet. Der Mediansplit ist ein gängiges statistisches Vorgehen zur Bildung dichotomer Variablen mit 2 gleich großen Gruppen. Darüber hinaus ist aus einer inhaltlichen Perspektive heraus anzunehmen, dass ein Cut-off von $134,52 \mathrm{~km}$ gut zwischen wohnortnaher und -ferner Behandlung unterscheidet. Im zweiten Schritt wurden zur Überprüfung von Gruppenunterschieden in den 2 Gruppen «wohnortnah» und «wohnortfern» bei kategorialen Variablen Chi-Quadrat-Tests und bei kontinuierlichen Variablen t-Tests durchgeführt. Aufgrund der Durchführung von 13 Tests wurde das Alpha-Niveau Bonferroni-korrigiert und auf 0,004 festgelegt. Im dritten Schritt wurde unter Einschluss von Störvariablen als Zwischensubjektfaktoren oder Kovariaten je eine Messwiederholungs-Varianzanalyse (ANOVA) für Depressivität, Ängstlichkeit und Somatisierung als abhängige Variable mit dem Messwiederholungsfaktor Zeit (Aufnahme, Entlassung, 6-Monats-Katamnese) und dem Zwischensubjektfaktor Distanz zum Wohnort (wohnortnah, wohnortfern) berechnet. Auch hier wurde das Alpha-Level wegen der 3 durchgeführten Tests Bonferroni-korrigiert und auf 0,02 festgelegt. Effektstärken werden als partielles Eta-Quadrat berichtet. Hierbei repräsentieren Werte > 0,01 kleine Effekte, > 0,06 mittlere Effekte und > 0,14 große Ef- 
Tab. 1. Soziodemobehandlungsspezifische Merkmale der Stichprobe grafische, klinische und

\begin{tabular}{|c|c|c|c|c|c|}
\hline Merkmal & $\begin{array}{l}\text { Gesamt } \\
(\mathrm{N}=1959)\end{array}$ & $\begin{array}{l}\text { Wohnortnah } \\
(\leq 134,52 \mathrm{~km}) \\
(\mathrm{n}=979)\end{array}$ & $\begin{array}{l}\text { Wohnortfern } \\
(\geq 134,53 \mathrm{~km}) \\
(\mathrm{n}=979)\end{array}$ & $\begin{array}{l}\text { Test- } \\
\text { statistik }\end{array}$ & $\mathrm{p}$ \\
\hline Geschlecht, $n(\%)$ weiblich, $\chi^{2}$ & $1189(60,8)$ & $591(60,4)$ & $597(61,1)$ & 0,11 & 0,74 \\
\hline Alter, $M(S D), \mathrm{t}$ & $48,23(11,56)$ & $47,33(12,02)$ & $49,13(11,03)$ & 3,47 & 0,001 \\
\hline Beziehung, $n(\%)$ ja, $\chi^{2}$ & $466(30,4)$ & $258(31,5)$ & $208(29,0)$ & 1,16 & 0,28 \\
\hline Kinder, $\mathrm{n}(\%) \mathrm{ja}, \chi^{2}$ & $896(58,4)$ & $473(57,8)$ & $423(59,0)$ & 0,22 & 0,64 \\
\hline Bildungsstand, $\mathrm{n}(\%), \chi^{2}$ & & & & 11,22 & 0,01 \\
\hline Sonder-/Hauptschulabschluss & $225(15,7)$ & $140(18,5)$ & $85(12,6)$ & & \\
\hline Realschulabschluss & $542(37,8)$ & $288(38,0)$ & $254(37,7)$ & & \\
\hline (Fach-)Abitur & $644(45,0)$ & $318(42,0)$ & $326(48,4)$ & & \\
\hline Sonstiges & $21(1,5)$ & $12(1,6)$ & $9(1,3)$ & & \\
\hline Komorbidität, $\mathrm{n}(\%) \mathrm{ja}, \chi^{2}$ & $1025(52,3)$ & $560(57,2)$ & $465(47,5)$ & 18,48 & $<0,001$ \\
\hline Affektive Störungen & $121(6,2)$ & $78(8,0)$ & $43(4,4)$ & & \\
\hline Angststörungen & $469(23,9)$ & $257(26,3)$ & $212(21,7)$ & & \\
\hline Somatoforme Störungen & $373(19,0)$ & $194(19,8)$ & $179(18,3)$ & & \\
\hline Essstörungen & $114(5,8)$ & $63(6,4)$ & $51(5,2)$ & & \\
\hline Persönlichkeitsstörungen & $234(11,9)$ & $143(14,6)$ & $91(9,3)$ & & \\
\hline Substanzbezogene Störungen & $117(6,0)$ & $69(7,0)$ & $48(4,9)$ & & \\
\hline \multicolumn{6}{|l|}{ Prä-Symptomschwere, $\mathrm{M}(\mathrm{SD}), \mathrm{t}$} \\
\hline PHQ - Depressivität & $14,63(5,54)$ & $15,02(5,51)$ & $14,25(5,54)$ & $-3,07$ & 0,002 \\
\hline PHQ - Ängstlichkeit & $11,35(4,78)$ & $11,50(4,76)$ & $11,19(4,81)$ & $-1,42$ & 0,16 \\
\hline PHQ - Somatisierung & $12,78(5,19)$ & $12,91(5,25)$ & $12,63(5,13)$ & $-1,20$ & 0,23 \\
\hline Behandlungsdauer, $\mathrm{M}(\mathrm{SD}), \mathrm{t}$ & $46,55(13,58)$ & $46,36(12,50)$ & $46,74(14,59)$ & 0,62 & 0,54 \\
\hline Antidepressive Begleitbehandlung, $n(\%)$ ja, $\chi^{2}$ & $966(49,3)$ & $538(55,0)$ & $428(43,7)$ & 24,72 & $<0,001$ \\
\hline Anzahl stationärer Vorbehandlungen, $\mathrm{M}$ (SD), $\mathrm{t}$ & $0,70(1,19)$ & $0,70(1,19)$ & $0,69(1,20)$ & $-0,18$ & 0,86 \\
\hline Klinik, $\chi^{2}$ & $1959(100,0)$ & $979(99,9)$ & $979(99,9)$ & 191,06 & $<0,001$ \\
\hline
\end{tabular}

fekte [Cohen, 1988]. Die Berechnungen wurden neben dem Median als Cut-off $(134,5 \mathrm{~km})$ auch mit einem Cut-off von $50 \mathrm{~km}$ durchgeführt, da dieser für manche Personengruppen möglicherweise noch besser zwischen wohnortnaher und -ferner Behandlung differenziert. Zum Schluss wurden potenzielle Zusammenhänge zwischen der Entfernung des Wohnorts zur Klinik und dem Therapieergebnis mittels der Berechnung von 3 Pearson-Korrelationen mit der kontinuierlichen Variable Distanz zur Klinik in Kilometern sowie den mittleren Veränderungen der Depressivität, Ängstlichkeit sowie Somatisierung von der Aufnahme bis zum Katamnesezeitpunkt ermittelt.

\section{Ergebnisse}

\section{Vorausgehende Analysen}

Die vorausgehenden Analysen zur Testung der soziodemografischen, klinischen und behandlungsspezifischen Variablen auf Unterschiede in den Gruppen "wohnortfern» und "wohnortnah» ergaben signifikante Unterschiede für die Variablen Alter $(\mathrm{t}(1942)=3,47 ; \mathrm{p}=0,001)$, Ausgangsdepressivität $(\mathrm{t}(1956)=-3,07 ;$ $\mathrm{p}=0,002)$, Komorbidität $\left(\chi^{2}(1)=18,48 ; \mathrm{p}<0,001\right)$, antidepressive Begleitbehandlung $\left(\chi^{2}(1)=24,72 ; \mathrm{p}<0,001\right)$ und Klinik $\left(\chi^{2}(6)=\right.$ 191,06; $\mathrm{p}<0,001$ ) (Tab. 1). Deshalb wurde die kontinuierliche Variable Alter als Kovariate und die kategorialen Variablen Komorbidität, Antidepressiva und Klinik als zusätzliche Zwischensubjektfaktoren in alle 3 Messwiederholungs-ANOVA aufgenommen. Die Ausgangsdepressivität wurde nur bei der Untersuchung des Effekts der Distanz auf die Depressivität als Kovariate aufgenommen.

\section{Hauptanalysen}

Die Messwiederholungs-ANOVA mit dem Messwiederholungsfaktor Zeit und dem Gruppenfaktor Distanz zum Wohnort mit dem Median als Cut-off (134,5 km) ergab für die abhängige Variable Depressivität unter Kontrolle der Störvariablen Ausgangsdepressivität, Alter, Komorbidität, Antidepressiva und Klinik einen signifikanten Zeiteffekt $\left(\mathrm{F}(1 ; 1906)=8,57 ; \mathrm{p}=0,003\right.$; partielles $\left.\eta^{2}=0,004\right)$; die Zeit $\times$ Distanz-Interaktion $(\mathrm{F}(1 ; 1906)=0,54 ; \mathrm{p}=0,46$; partielles $\left.\eta^{2}<0,001\right)$ wurde nicht signifikant. Dies deutet darauf hin, dass die Behandlung über die 3 Messzeitpunkte hinweg effektiv zur Reduktion von Depressivität ist, sich jedoch nicht in Abhängigkeit von der Distanz zum Wohnort unterscheidet (Abb. 1). Die Dreifachinteraktion Zeit $\times$ Distanz $\times$ Klinik $(\mathrm{F}(6 ; 1906)=1,13 ; \mathrm{p}=0,34$; partielles $\left.\eta^{2}=0,004\right)$ wurde nicht signifikant, was bestätigt, dass die Klinik keinen moderierenden Einfluss auf eine potenzielle Zeit $x$ Distanz-Interaktion hat. Auch bei einem Cut-off von $50 \mathrm{~km}$ ergab eine Messwiederholungs-ANOVA mit dem Messwiederholungsfaktor Zeit und dem Gruppenfaktor Distanz zum Wohnort unter Kontrolle der Störvariablen Klinik, Bildungsstand und Antidepressiva zwar einen signifikanten Zeiteffekt $(\mathrm{F}(1,88 ; 2534,97)=134,02$; $\mathrm{p}<$ 0,001; partielles $\left.\eta^{2}=0,09\right)$, jedoch keine signifikante Distanz $\times$ ZeitInteraktion $\left(F(1,88 ; 2534,97)=1,18 ; p=0,31\right.$; partielles $\left.\eta^{2}=0,001\right)$. Dies bestätigt erneut, dass die Effektivität der Behandlung zur Reduktion der Depressivität nicht durch die Entfernung zwischen Klinik und Wohnort beeinflusst wird. 
Abb. 1. Mittelwerte und Standardfehler der Depressivität bei Aufnahme, Entlassung und katamnestisch 6 Monate später bei wohnortnaher und wohnortferner stationärer Behandlung.

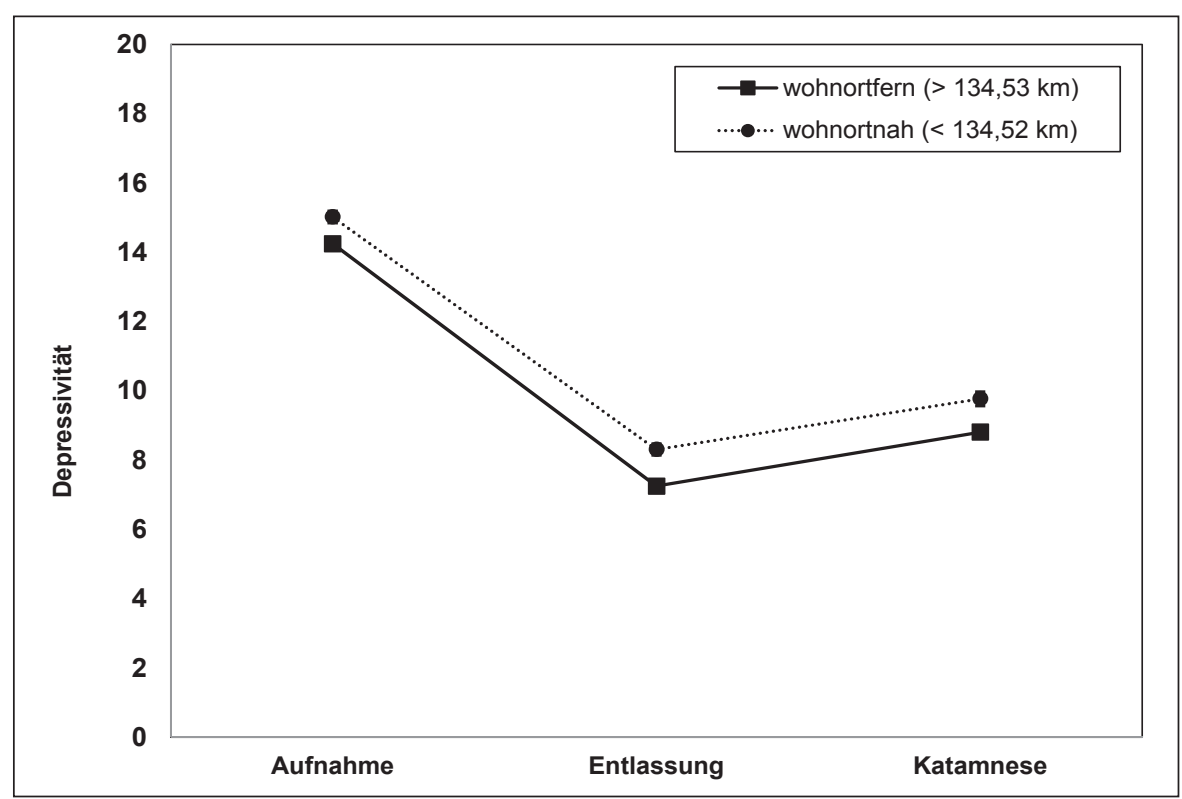

Die Messwiederholungs-ANOVA mit dem Messwiederholungsfaktor Zeit und dem Zwischensubjektfaktor Distanz mit dem Median als Cut-off (134,5 km) für die abhängige Variable Ängstlichkeit ergab unter Kontrolle der Störvariablen Alter, Komorbidität, Antidepressiva sowie Klinik ebenfalls einen signifikanten Zeiteffekt $\left(F(1,86 ; 3547,12)=50,02 ; p<0,001\right.$; partielles $\left.\eta^{2}=0,26\right)$, jedoch keine signifikante Interaktion $(\mathrm{F}(1,86 ; 3547,12)=1,62 ; \mathrm{p}=$ 0,20; partielles $\eta^{2}=0,001$ ). Auch in Bezug auf die Reduktion der Ängstlichkeit der Patienten über die Zeit hinweg scheint es also keine Unterschiede in Abhängigkeit von der Nähe der Klinik zum Wohnort zu geben. Auch hier wurde die Dreifachinteraktion Zeit $\times$ Distanz $\times$ Klinik $(\mathrm{F}(11,16 ; 3547,12)=1,65 ; \mathrm{p}=0,78$; partielles $\left.\eta^{2}=0,005\right)$ nicht signifikant. Darüber hinaus ergab auch bei einem Cut-off von $50 \mathrm{~km}$ eine Messwiederholungs-ANOVA mit dem Messwiederholungsfaktor Zeit und dem Gruppenfaktor Distanz zum Wohnort unter Kontrolle der Störvariablen Klinik, Bildungsstand und Antidepressiva zwar einen signifikanten Zeiteffekt $\left(\mathrm{F}(1,88 ; 2531,06)=100,69 ; \mathrm{p}<0,001 ;\right.$ partielles $\left.\eta^{2}=0,07\right)$, jedoch keine signifikante Distanz $\times$ Zeit-Interaktion $(\mathrm{F}(1,88 ; 2531,06)=$ 0,12; $\mathrm{p}=0,87$; partielles $\eta^{2}<0,001$ ).

Bei der Messwiederholungs-ANOVA für das Outcome-Maß Somatisierung zeigte sich beim Median als Cut-off (134,5 km) unter Berücksichtigung der Störvariablen Alter, Komorbidität, Antidepressiva sowie Klinik ein signifikanter Zeiteffekt ( $\mathrm{F}(1,89$; $3598,97)=43,90 ; p<0,001$; partielles $\left.\eta^{2}=0,02\right)$, jedoch kein signifikanter Interaktionseffekt $(\mathrm{F}(1,89 ; 3598,97)=1,00 ; \mathrm{p}=0,37$; partielles $\left.\eta^{2}=0,001\right)$. Dies bedeutet, dass die Veränderung der Somatisierungsbeschwerden der Patienten über die Zeit hinweg unabhängig von der Nähe der Klinik zum Wohnort ist. Die nichtsignifikante Dreifachinteraktion Zeit $\times$ Distanz $\times$ Klinik $(\mathrm{F}(11,32$; $3598,97)=0,55 ; \mathrm{p}=0,88$; partielles $\left.\eta^{2}=0,002\right)$ zeigt, dass dieser Effekt nicht über die Klinik moderiert wird. Auch bei einem Cut-off von $50 \mathrm{~km}$ ergab die entsprechende Messwiederholungs-ANOVA unter Kontrolle der Störvariablen Klinik, Bildungsstand und Anti- depressiva wieder einen signifikanten Zeiteffekt $(\mathrm{F}(1,88 ; 2541,11)=$ 42,66; $p<0,001$; partielles $\left.\eta^{2}=0,03\right)$, jedoch keine signifikante Zeit $\times$ Entfernung-Interaktion $(F(1,88 ; 2541,11)=0,69 ; p=0,49$; partielles $\eta^{2}=0,001$ ).

Übereinstimmend mit den zuvor aufgeführten Ergebnissen ergaben die 3 Korrelationen zwischen der Entfernung des Wohnorts zur Klinik und den mittleren Veränderungen der Depressivität ( $\mathrm{r}=$ $-0,009 ; \mathrm{p}=0,70$; Abb. 2), Ängstlichkeit $(\mathrm{r}=-0,004 ; \mathrm{p}=0,85)$ sowie Somatisierung $(\mathrm{r}=-0,007 ; \mathrm{p}=0,74)$ über die Therapie und Katamnese hinweg keine signifikanten Zusammenhänge.

\section{Post-hoc-Analysen}

Um zu prüfen, ob die Befunde dadurch verzerrt wurden, dass bei 39,9\% der Patienten der PHQ bei Katamnese fehlte, wurde in 3 weiteren ANOVA getestet, ob das Therapie-Outcome durch die Distanz moderiert wird, wenn Alter, Ausgangsdepressivität (nur bei ANOVA mit Depressivität als Outcome), Komorbidität, Antidepressiva, Klinik und das Vorliegen der 6-Monats-Katamnese als Kovariaten berücksichtigt werden und nur die Symptomveränderungen zwischen Aufnahme und Entlassung untersucht werden. Die Analysen ergaben jedoch unabhängig davon, ob die Katamnese vorlag oder nicht, weder für die Depressivität $(F(1 ; 3158)=0,07$; $\mathrm{p}=0,92$; partielles $\left.\eta^{2}<0,001\right)$ noch für die Ängstlichkeit $(\mathrm{F}(1$; $3159)=0,74 ; p=0,39$; partielles $\left.\eta^{2}<0,001\right)$ noch für die Somatisierungsbeschwerden $\left(\mathrm{F}(1 ; 3159)=0,31 ; \mathrm{p}=0,58\right.$; partielles $\eta^{2}=$ $0,001)$ signifikante Unterschiede zwischen wohnortnah und -fern behandelten Patienten. Somit können Verzerrungen durch die selektive Stichprobe in Bezug auf das Behandlungsergebnis ausgeschlossen werden. Darüber hinaus zeigten sich zwischen den $\mathrm{Pa}$ tienten mit und ohne Katamnesedaten keine Unterschiede in den Korrelationen zwischen der Entfernung und den mittleren Symptomveränderungen zwischen Aufnahme und Entlassung (Depressivität: $\mathrm{z}=0,56, \mathrm{p}=0,29$; Ängstlichkeit: $\mathrm{z}=1,20, \mathrm{p}=0,11$; Somatisierung: $z=-0,31, p=0,38$ ). 
Abb. 2. Streudiagramm für den Zusammenhang zwischen der Entfernung Wohnort-Klinik und der Depressivitätsabnahme zwischen Aufnahme und Katamnese.

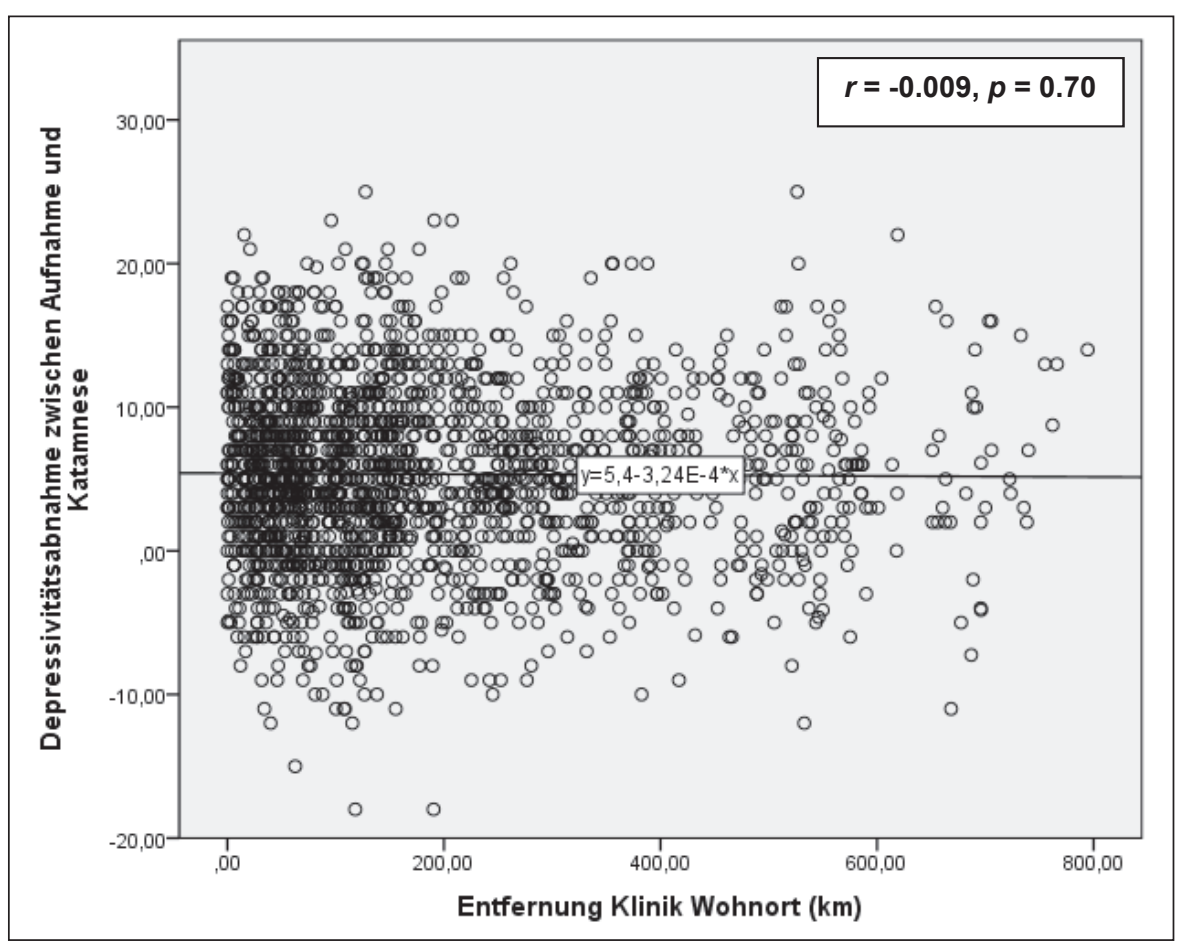

\section{Diskussion}

Die Ergebnisse der vorliegenden Studie weisen nicht darauf hin, dass bei stationärer Behandlung von Patienten mit depressiven Erkrankungen die Entfernung zwischen Wohnort und Klinik einen Einfluss auf das Behandlungsergebnis hat. $\mathrm{Zu}$ einem ähnlichen Ergebnis kamen Funke und Missel [2009], die ebenso keinen Einfluss auf das Therapie-Outcome, in diesem Fall bei Patienten mit Abhängigkeitserkrankungen, fanden. Dieses Ergebnis mag überraschend erscheinen, da viele Autoren [Hell, 2002; Fritze et al., 2001; Hohagen, 2002, 2006] eine wohnortnahe Behandlung als vorteilhaft ansehen, weil davon ausgegangen wird, dass eine damit assoziierte bessere soziale Einbindung und Anschlussbehandlung das Therapieergebnis positiv beeinflussen. Andere Autoren [Fritze et al., 2001; Funke und Missel, 2009] weisen jedoch auch auf die Vorteile der wohnortfernen Behandlung hin, nämlich beispielsweise den einer diskreten Behandlung in oftmals ländlich gelegenen, spezialisierten Fachkliniken. Wir vermuten aufgrund der vorliegenden Ergebnisse, dass die Distanz zwischen Klinik und Wohnort per se keinen entscheidenden Einfluss auf den kurz- und langfristigen Therapieerfolg hat.

Die vorliegende Studie hat einige Stärken und Schwächen. Als Stärken können das Einbeziehen mehrerer Kliniken, die große klinische Stichprobe sowie das Vorliegen eines Katamnesezeitpunkts angesehen werden. Als einschränkend zu betrachten ist der fehlende Einsatz eines strukturierten Interviews zur Validierung der Diagnosen (z.B. das Strukturierte Klinische Interview für DSM-IV [Wittchen et al., 1997]) sowie die Dichotomisierung der kontinuierlichen Variable Distanz zum Wohnort in Kilometern, die häufig kritisch betrachtet wird, da dies zu einer Reduktion der Teststärke und damit einer konservativeren Signifikanzaussage führen kann [Cohen, 1983]. Relativierend zu diesem Kritikpunkt ist jedoch zu sagen, dass die Korrelationen zwischen den kontinuierlichen Variablen Distanz zum Wohnort in Kilometern und den mittleren Veränderungen in Depressivität/Ängstlichkeit/Somatisierung ebenso keinen Zusammenhang zwischen Entfernung und Symptombesserung nachweisen konnten. Der größte Kritikpunkt der vorliegenden Studie besteht jedoch darin, dass man aus den vorliegenden Befunden zwar den Schluss ziehen kann, dass es keinen Unterschied zwischen wohnortferner und -naher Behandlung gemessen mit der räumlichen Distanz zwischen Klinik und Wohnort gibt, jedoch nicht spezifiziert werden kann, welche Rolle die in der Diskussion um die Vorteile beider Behandlungsalternativen genannten Behandlungsspezifika, wie die Spezialisierung der Behandlung sowie die Anschlussbehandlung, dabei spielen. Beispielsweise konnten wir mit unserer Untersuchung weder überprüfen, ob die wohnortferne Behandlung der wohnortnahen tatsächlich überlegen ist, weil sie eine größere Spezialisierung bietet, noch die Hypothese, dass die wohnortnahe der wohnortfernen Behandlung aufgrund besserer Anschlussbehandlungsmöglichkeiten überlegen ist; denn durch den selektiven Einschluss von Patienten aus den Schön Kliniken war die starke Spezialisierung der Behandlung in beiden Bedingungen (wohnortnah und wohnortfern) gegeben und die hausinternen Anschlussbehandlungsmöglichkeiten wurden in keiner der beiden Bedingungen realisiert.

In zukünftigen Studien sollte man neben dem Einfluss der faktischen räumlichen Distanz auch den der subjektiv empfundenen Distanz erfassen, denn was tatsächlich als wohnortnah oder -fern empfunden wird, ist höchstwahrscheinlich subjektiv. Während für 
den einen Patienten eine Distanz von $130 \mathrm{~km}$ wohnortnah ist, ist sie für den anderen wohnortfern. Dies mag von unterschiedlichen Faktoren wie beispielsweise den Beförderungsmöglichkeiten sowie der Lage des Wohnorts abhängen. Darüber hinaus sollte in zukünftigen Studien untersucht werden, welche Rolle die in der Diskussion um die wohnortnahe bzw. -ferne Versorgung angeführten inhaltlichen Aspekte tatsächlich für den Behandlungserfolg wohnortnaher und -ferner Behandlung spielen, insbesondere vor dem Hintergrund, dass es Hinweise darauf gibt, dass der Spezialisierungsgrad der Behandlungseinrichtung sowie die Intensität der Arbeit an der Umsetzung individueller Nachsorgemaßnahmen wichtig zu sein scheinen [z.B. Ebert et al., 2013; Fontana und Rosenheck, 1997; Gönner et al., 2006; Golkaramnay et al., 2007; Schramm et al., 2007]. Die Hypothese, dass die wohnortnahe der wohnortfernen Behandlung aufgrund besserer Anschlussbehandlungsmöglichkeiten überlegen ist, könnte beispielsweise in einer kontrollierten Studie überprüft werden, in der man die Effekte einer stationären Behandlung mit hausinterner Anschlussbehandlung mit denen einer stationären Behandlung ohne eine solche Anschlussbehandlung vergleichen würde. Unseres Wissens liegen solche wissenschaftlichen Untersuchungen bisher noch nicht vor. Darüber hinaus wäre es interessant zu erfassen, welchen Einfluss die Gründe für die Auswahl einer Klinik auf den Erfolg wohnortnaher und -ferner Behandlungen haben. Wenn man davon ausgeht, dass sich ein Patient, der auf dem Land lebt, eher eine ländlich gelegene, wohnortnahe Klinik aussucht als eine städtisch gelegene, wohnortferne, sollte man zudem auch den Einfluss der Lage des Wohnorts (Stadt vs. Land) erfassen. Schließlich sollten potenziell moderierende Einflüsse von Persönlichkeitsvariablen in Modera- toranalysen untersucht werden, da sich wahrscheinlich für unterschiedliche Patienten auch unterschiedliche Behandlungssettings, sprich wohnortnah oder auch -fern gelegene Behandlungsinstitutionen, eignen.

Die Überprüfung solcher Fragestellungen wäre auch vor dem Hintergrund des gesundheitspolitischen Propagierens wohnortnaher Konzepte sinnvoll. Die wohnortnahe Versorgung mit hausinterner Anschlussbehandlung mag in bestimmten Fällen (z.B. bei Patienten mit Psychose) besonders indiziert sein, und eine Weiterbehandlung durch denselben Therapeuten bietet sicherlich Vorteile. Gleichzeitig sollte aber auch berücksichtigt werden, dass dies auch in Institutionen mit eigener Tagesklinik und Ambulanz oft nicht möglich ist und dass eine flächendeckende wohnortnahe Behandlung in städtischen Kliniken auf Kosten spezialisierter Therapieangebote gehen könnte. Eine flächendeckende wohnortnahe Versorgung könnte dazu führen, dass an städtischen Krankenhäusern viele kleine Abteilungen etabliert werden würden, die allein aufgrund ihrer Größe keine hohen Fallzahlen und somit auch keinen hohen Spezialisierungsgrad für bestimmte Krankheitsbilder erreichen könnten. Als Konsequenz würde kein breites therapeutisches und fachtherapeutisches Angebot etabliert und auch kein entsprechend spezialisiertes Personal ausgebildet werden, was zu Lasten der Qualität der Behandlung gehen könnte.

\section{Disclosure Statement}

Es liegen keine Interessenkonflikte vor.

\section{Literatur}

APA (American Psychiatric Association): Diagnostic and Statistical Manual of Mental Disorders DSM-IV-TR. Washington, DC, American Psychiatric Association, 2000.

Arolt V, Reimer C, Dilling H: Soziotherapie, Versorgung, \& Rehabilitation; in Arolt V, Reimer C, Dilling $\mathrm{H}$ (Hrsg): Basiswissen Psychiatrie und Psychotherapie. Berlin, Heidelberg, Springer, 2011, pp 363-379.

Busch MA, Maske UE, Ryl L, et al: Prävalenz von depressiver Symptomatik und diagnostizierter Depression bei Erwachsenen in Deutschland - Ergebnisse der Studie zur Gesundheit Erwachsener in Deutschland (DEGS1). Bundesgesundheitsblatt 2013;56:733-739.

Cohen J: The cost of dichotomization. Appl Psychol Meas 1983;7:249-253.

Cohen J: Statistical Power Analysis for the Behavioral Sciences. Hillsdale, NJ, Erlbaum, 1988.

DGPPN, BÄK, KBV, AWMF, AkdÄ, BPtK, BApK, DAGSHG, DEGAM, DGPM, DGPs, DGRW (Hrsg) für die Leitliniengruppe Unipolare Depression: S3-Leitlinie/ Nationale VersorgungsLeitlinie Unipolare DepressionKurzfassung, ed 1. Berlin, Düsseldorf, 2009.

Dilling H, Mombour W, Schmidt MH (Hrsg): Internationale Klassifikation psychischer Störungen, ICD-10 Kapitel V. Bern, Huber, 1993.
Ebert D, Tarnowski T, Gollwitzer M, et al: A transdiagnostic Internet-based maintenance treatment enhances the stability of outcome after inpatient cognitive behavioral therapy: a randomized controlled trial. Psychother Psychosom 2013;82:246-256.

Fontana A, Rosenheck R: Effectiveness and cost of the inpatient treatment of posttraumatic stress disorder: comparison of three models of treatment. Am J Psychiatry 1997;154:758-765.

Fritze J, Schmauß M, Saß H für den Vorstand der Deutschen Gesellschaft für Psychiatrie, Psychotherapie und Nervenheilkunde (DGPPN): Die Krankenhausplanung der Länder muss Ausbau der gemeindenahen Psychiatrie im Sinne der Entstigmatisierung vorantreiben. Nervenarzt 2001;2:327-328.

Funke W, Missel P: Gemeindenahe und gemeindeferne Behandlung: empirische Hinweise zur Indikationsstellung. Sucht AKTUELL 2009;2:35-38.

Golkaramnay V, Bauer S, Haug S, et al: The exploration of the effectiveness of group therapy through an Internet chat as aftercare: a controlled naturalistic study. Psychother Psychosom 2007;76:219-225.

Gönner S, Bischoff C, Ehrhardt M, et al: Effekte therapiezielorientierter kognitiv-verhaltenstherapeutischer Nachsorgemaßnahmen auf den Therapietransfer im Anschluss an eine stationäre psychosomatische Rehabilitationsbehandlung. Die Rehabilitation 2006;45: 369-376.
Gräfe K, Zipfel S, Herzog W, et al: Screening psychischer Störungen mit dem «Gesundheitsfragebogen für Patienten (PHQ-D)». Diagnostica 2004;50:171-181.

Hell D: Gemeinschaft und psychische Krankheit. Schweiz Arch Neurol Psychiatr 2002;153:37-39.

Hohagen F: Ist die Psychotherapie in der Psychiatrie gefährdet? Psychother Psychosom Med Psychol 2002;52: 3-4.

Hohagen F: Die Deklaration von Helsinki - eine Herausforderung für die deutsche Psychiatrie. Nervenarzt 2006;77:1133-1142.

Hohagen F: Privatisierung in Psychiatrie und Psychotherapie - zwischen Shareholder Value und öffentlichrechtlicher Gesundheitsfürsorge. Nervenarzt 2007;78: 1082-1094.

Kroenke K, Spitzer RL, Williams JB: The PHQ-9: validity of a brief depression severity measure. J Gen Intern Med 2001;16:606-613.

Kroenke K, Spitzer R, Williams JB: The PHQ-15: validity of a new measure for evaluating the severity of somatic symptoms. Psychosom Med 2002;64:258-266.

Lépine JP, Briley M: The increasing burden of depression. Neuropsychiatr Dis Treat 2011;7:3-7.

Löwe B, Spitzer RL, Zipfel S, et al: PHQ-D - Gesundheitsfragebogen für Patienten. Manual - Komplettversion und Kurzform. www.klinikum.uni-heidelberg.de/fileadmin/ Psychosomatische_Klinik/download/PHQ_Manual1. pdf, 2002. 
Löwe B, Unützer J, Callahan CM, et al: Monitoring depression treatment outcomes with the Patient Health Questionnaire-9. Med Care 2004;42:1194-1201.

Löwe B, Decker O, Müller S: Validation and standardization of the Generalized Anxiety Disorder Screener (GAD-7) in the general population. Med Care 2008;46: 266-274.

Rohde P, Lewinsohn PM, Seeley JR: Comorbidity of unipolar depression: II. Comorbidity with other mental disorders in adolescents and adults. J Abnorm Psychol 1991;100:214-222.

Schramm E, van Calker D, Dykierek P, et al: An intensive treatment program of interpersonal psychotherapy plus pharmacotherapy for depressed inpatients: acute and long-term results. Am J Psychiatry 2007;164:768777.
Schulz H, Barghaan DPD, Koch U, et al: Die Versorgung von Patienten mit psychischen Störungen; in Wittchen HU, Hoyer J (Hrsg): Klinische Psychologie und Psychotherapie. Berlin, Heidelberg, Springer, 2011, pp 361380.

Spitzer RL, Kroenke K, Williams JB, et al: A brief measure for assessing generalized anxiety disorder: the GAD-7. Arch Intern Med 2006;166:1092-1097.

Titov N, Dear BF, McMillan D, et al: Psychometric comparison of the PHQ-9 and BDI-II for measuring response during treatment of depression. Cogn Behav Ther 2011;40:126-136

Wittchen HU, Zaudig M, Fydrich T: Strukturiertes Klinisches Interview für DSM-IV. Göttingen, Hogrefe, 1997.
Wittchen HU, Jacobi F, Klose M, et al: Depressive Erkrankungen; in Robert Koch-Institut (Hrsg): Gesundheitsberichterstattung des Bundes. Berlin, Robert Koch-Institut, 2010, Heft 51.

Zingg C, Schmidt P, Küfner H, et al: Der Zusammenhang zwischen Selbstwirksamkeit und Alkoholabstinenz bei ambulant und stationär behandelten Patienten: Ergebnisse einer 2-Jahres-Katamnese. Psychosom Med Psychol 2009;59:307-313. 